\title{
Média móvel e a Curva de Phillips: previsões para a taxa de inflação em uma amostra de países desenvolvidos e em desenvolvimento
}

Moving Average and the Phillips Curve: forecasts for the inflation rate in a sample of developed and developing countries

\author{
Erika Vanessa Alves da Silva (1) \\ Nathália da Silva Oliveira (2) \\ Roberto Tatiwa Ferreira ${ }^{(3)}$ \\ Cristiano da Costa da Silva (4)
}

\author{
(1) Universidade Federal do Ceará \\ (2) Universidade Federal do Ceará \\ (3) Universidade Federal do Ceará \\ (4) Universidade do Estado do Rio \\ Grande do Norte
}

\begin{abstract}
This study evaluates the inflation forecasts produced by Phillips curve models with and without ARMA modeling of their errors, considering a sample that contains developed and developing countries. The aim of this study is to provide empirical evidence that this simple reformulation of the Phillips curve can serve as a benchmark for studies that propose econometric or time series models more elaborated to predict the rate of inflation. The results show that the use of ARMA components in the Phillips curve decrease considerably its mean square error of forecast for all countries in the sample.
\end{abstract}

\section{Keywords}

inflation; forecasting; Phillips Curve.

JEL Codes C22; C53; E31; E37.

\section{Resumo}

Este trabalho avalia a eficiência preditiva dos modelos de curva de Phillips com e sem modelagem ARMA dos seus resíduos, considerando uma amostra que contém países desenvolvidos e em desenvolvimento. O objetivo principal deste estudo é fornecer evidências empiricas de que essa simples reformulação da Curva de Phillips pode servir de benchmark para estudos que propõem modelos econométricos ou de séries temporais mais elaborados para prever a taxa de inflação. Os resultados mostram que o uso de componentes ARMA nos resíduos da Curva de Phillips diminuem consideravelmente seus erros quadrados médios de previsão para todos os países da amostra.

\section{Palavras-chave}

inflação; previsão; Curva de Phillips.

Códigos JEL C22; C53; E31; E37. 


\section{Introdução}

A inflação consiste no aumento contínuo e generalizado dos preços nominais que ocorre tanto em países desenvolvidos quanto subdesenvolvidos. Esse processo gera vários efeitos negativos, entre os quais destacam-se a possibilidade de um nível de crescimento abaixo do ótimo e do aumento da pobreza em um país. Com a instabilidade nos preços, os agentes econômicos (famílias, empresas e governo) devem tomar suas decisões com um menor horizonte de planejamento e surgem distorções entre os preços relativos dos diversos setores da economia. Por outro lado, a inflação age como um imposto que atinge com maior impacto as famílias com menor poder aquisitivo. Por essas razões, a inflação é uma instabilidade econômica monitorada pelos agentes econômicos e combatida pelos bancos centrais, os quais precisam, nesses processos, de previsões dessa variável para formar expectativas sobre o comportamento futuro do nível de preços da economia, auxiliando-os nas suas decisões no presente e no futuro.

As previsões de inflação tornam-se ainda mais importantes em economias que utilizam metas inflacionárias, como o Brasil a partir de 1999. Nesse mesmo ano foi criado o Departamento de Estudos e Pesquisas (Depep) que, entre outras funções, é responsável pelas previsões da inflação realizadas pelo Banco Central (BC) brasileiro (Lima et al., 2011).

Dada a importância de se prever a taxa de inflação, metodologias diversas são utilizadas com esse objetivo e suas habilidades preditivas são comparadas. Uma comparação usual é a de previsões geradas por modelos de séries temporais, como por exemplo modelos autorregressivos de média móvel (ARMA) e de modelos vetoriais autorregressivos (VAR), versus as previsões de modelos estruturais, como por exemplo modelos baseados na Curva de Phillips e modelos dinâmicos estocásticos de equilíbrio geral dinâmico (DSGE).

Essas metodologias também são utilizadas pelo Banco Central brasileiro. De acordo com Lima et al. (2011), os modelos do BC para essa finalidade são classificados em: i) modelos de indicadores antecedentes e núcleos de inflação; ii) modelos VAR; iii) modelos semiestruturais pequenos; iv) modelos médios; e v) modelos microfudamentados de médio porte (DSGE). A curva de Phillips é uma das equações mais importantes dos modelos semiestruturais de pequeno porte e também pode fazer parte dos modelos VAR estruturais, médios e DSGE, representando as condições de oferta agregada da economia. 
Dessa forma, visando contribuir com evidências empíricas sobre essa questão, o objetivo principal deste estudo consiste em avaliar a eficiência preditiva dos modelos de Curva de Phillips com e sem modelagem ARMA dos seus resíduos, considerando uma amostra que contém países desenvolvidos e em desenvolvimento. Vale ressaltar que o objetivo deste trabalho não é o de escolher um melhor modelo para prever a inflação, e sim de apresentar evidências de que os trabalhos nessa área podem chegar a conclusões diferentes sobre a eficiência das previsões de inflação geradas pelo modelo de Curva de Phillips ao não se utilizar modelos ARMA para os seus resíduos. Por essas razões, as previsões desse modelo serão comparadas com os modelos "tradicionais" ${ }^{1}$ de séries temporais utilizados para este propósito, isto é, modelos ARMA e VAR.

Apesar dos trabalhos de Blanchflower e Oswald (1994) e Roberts (1997) derivarem uma Curva de Phillips com erros na forma de média móvel, resultado que aponta para a possibilidade de modelagens ARMA dos resíduos da curva de Phillips serem necessárias, poucos trabalhos investigam se essa abordagem "híbrida" de modelo estrutural com uma modelagem de séries temporais para os seus resíduos pode gerar melhores previsões do que as sem considerar esses processos.

Os trabalhos que utilizam as previsões de inflação baseadas em modelos de Curva de Phillips usualmente utilizam sua versão tradicional, a qual inclui uma medida de expectativa de inflação, geralmente adaptativa, uma medida de "aquecimento da economia", como por exemplo o hiato do produto ou do desemprego e, alguns poucos, adicionam outras variáveis econômicas. Por exemplo, Rumler e Valderrama (2008) mostram que modelos da Curva de Phillips geram previsões mais eficientes para a inflação austríaca do que modelos univariados de séries temporais. Stock e Watson (1999) comparam as previsões para a inflação dos EUA geradas por modelos baseados na Curva de Phillips com as previsões de diversos modelos de séries temporais e outros especificados a partir da teoria econômica, com a inserção da taxa de juros, moeda e preços de mercadorias. Seus resultados indicam que a Curva de Phillips é um método robusto para previsão

1 Há vários modelos que incluem diversas não linearidades, como por exemplo efeitos limiares (threshold) ou de mudanças nos seus parâmetros, que incluem informações a priori via vetores autorregressivos Bayesianos (BVAR), ou que apresentam algum tipo de redução de seus parâmetros sem diminuir o conjunto de informação como no caso dos modelos de fatores. A eficiência preditiva desses modelos usualmente é comparada com a dos modelos ARMA e VAR sem essas características. 
da inflação. Stock e Watson (2003) reportam que para diferentes países os modelos autorregressivos univariados produzem as melhores previsões para a taxa de inflação do que modelos que incluem o preço de ativos ou a taxa de juros.

Em outro trabalho, Stock e Watson (2007) reportam que um modelo de média móvel integrada com parâmetros que variam no tempo gera as melhores previsões para inflação dos EUA. Esses autores, após decomporem a taxa de inflação em tendência estocástica e em um componente transitório, verificam que os modelos baseados na Curva de Phillips tendem a gerar melhores previsões nos períodos em que a variância do componente permanente é alta e a volatilidade do componente transitório se mostra estável. Quando o componente transitório apresenta maior variabilidade com um termo permanentemente estável, os modelos univariados usualmente geram as melhores previsões para a inflação dos EUA. O trabalho de Canova (2007) avalia a performance de previsão de inflação dos países do G-7, comparando as previsões geradas por modelos AR, VAR, VAR Bayesianos (BVAR), com fatores com parâmetros variantes no tempo e de Curva de Phillips. Seus resultados mostram que modelos baseados na curva de Phillips, melhoram a previsão em relação aos modelos univariados apenas nos horizontes longos de previsão, mas não para um passo à frente. As previsões dos modelos BVAR em média não são melhores do que as previsões geradas pelos modelos $A R$, e as do VAR são em média piores.

Para a economia brasileira, Sachsida (2013) faz uma revisão sobre trabalhos que estimam modelos de Curva de Phillips para o Brasil. Há vários trabalhos que reportam uma correlação significante do ponto de vista estatístico entre a inflação e a medida de excesso de demanda utilizada (hiato do produto, emprego ou custo marginal), corroborando a relação entre essas variáveis previstas pelo modelo em questão. Esse resultado é reportado nos estudos de Portugal e Madalozzo (2000), Correa e Minella (2005), Schwartzman (2006), Areosa e Medeiros (2007) e Mazali e Divino (2010). Por outro lado, há vários estudos que refutam essa relação como, por exemplo, os de Cysne (1985), Sachsida, Ribeiro, e Santos (2009) e Maka e Barbosa (2013). Por fim, há trabalhos que encontram uma relação significante entre essas variáveis apenas num determinado período de tempo, ou ainda, em um determinado regime, como em Arruda, Ferreira e Castelar (2011) e Mendonça et al. (2012). 
No que se refere à eficiência preditiva, objeto deste trabalho, Arruda, Ferreira e Castelar (2011) mostram que um modelo VAR produz menor erro quadrático médio de previsão (EOM) da taxa de inflação mensal brasileira, quando comparado aos modelos de Curva de Phillips lineares e modelos ARMA. Mas a introdução de efeitos limiares na Curva de Phillips faz com que este modelo gere um EQM 20\% menor do que o VAR. Ferreira (2014) estima uma curva de Phillips generalizada, no sentido de analisar a capacidade preditiva de vários previsores. Seus resultados indicam que a eficiência preditiva da Curva de Phillips depende do horizonte de previsão e das variáveis utilizadas (desemprego, taxa de câmbio, capacidade industrial utilizada, entre outras). Ferreira e Palma (2015) utilizam as técnicas dynamic model avareging e dynamic model selection e mostram que as especificações com um conjunto de regressores relacionados com a Curva de Phillips não geram as melhores previsões no curto e no longo prazo.

Um ponto em comum entre os estudos que comparam a eficiência preditiva da Curva de Phillips é a não inclusão de termos de média móvel, ou até mesmo autorregressivos nos seus resíduos. Esse problema pode ser reduzido ao se introduzir um maior número de defasagens da inflação para prever a inflação futura. Para isso pode ser necessário um grande número adicional de parâmetros na especificação da Curva de Phillips, principalmente quando a inflação exibe termos de média móvel. Entretanto, sabe-se que um dos componentes do erro de previsão é a falta de parcimônia, ou seja, modelos com maior número de parâmetros a serem estimados tendem a apresentar maiores erros de previsão.

As evidências empíricas desse estudo sugerem que há ganhos consideráveis na eficiência preditiva dos modelos de Curva de Phillips ao se introduzir uma modelagem ARMA nos seus erros. Tal resultado é esperado em virtude das séries de preços exibirem usualmente termos de média móvel. Assim, essa simples modificação da Curva de Phillips pode ser utilizada como benchmark nos trabalhos que propõem modelos econométricos ou de séries temporais mais elaborados para prever a taxa de inflação.

Além desta introdução, o presente estudo é composto por mais quatro seções. Na próxima seção, apresentam-se os principais aspectos teóricos, bem como os modelos econométricos utilizados. A terceira dedica-se à exposição dos dados. Os resultados da previsão da taxa de inflação para os grupos de países desenvolvidos e em desenvolvimento estão reportados na quarta seção. Por fim, temos as conclusões. 


\section{Modelos utilizados para a previsão da inflação}

\subsection{Modelos ARMA e VAR}

Modelos autorregressivos e de médias móveis (ARMA) univariados são muito utilizados para realizar previsões nas mais diversas áreas, inclusive na economia. Em virtude da sua simplicidade e da qualidade de suas previsões, esses modelos são usualmente a base de comparação para outros, como modelos baseados na curva de Phillips. Pode-se citar vários trabalhos que evidenciam a qualidade das previsões de inflação proporcionadas por esse tipo de modelo. Stock e Watson (2007), ao compararem a previsão de inflação dos EUA obtida por vários modelos, observam que modelos multivariados mais sofisticados dificilmente melhoram as previsões de um modelo univariado. Em um trabalho para os países do G7, Canova (2007) reporta que as previsões de inflação no curto prazodos modelos do tipo $\mathrm{ARMA}^{2}$ são eficientes e difíceis de serem melhoradas. Um modelo $\operatorname{ARMA}(p, q)$ pode ser expresso da seguinte forma:

$$
y_{t}=\mu+\phi_{1} y_{t-1}+\ldots+\phi_{p} y_{t-p}+\varepsilon_{t}+\theta_{1} \varepsilon_{t-1}+\ldots+\theta_{q} \varepsilon_{t-q}
$$

Nesse modelo, a variável dependente é explicada tanto pelas suas próprias defasagens como pelos valores passados dos choques. Geralmente um modelo com menor número de parâmetros é capaz de realizar melhores previsões do que outro modelo com maior número de variáveis e, portanto, de parâmetros. Esse princípio é chamado de parcimônia na área de previsão. Os modelos ARMA são usualmente parcimoniosos em virtude da sua capacidade de capturar a dinâmica da variável em análise com poucos parâmetros e gerarem previsões eficientes.

Por outro lado, é possível que informações adicionais sobre o futuro da inflação estejam contidas no presente ou no passado de outras variáveis. Nesse caso, espera-se que um vetor autoregressivo (VAR) gere melhores previsões do que os modelos ARMA. No trabalho de Canova (2007), modelos VAR Bayesianos geram, em média, as melhores previsões para a inflação dos países do G7, quando comparadas às produzidas pelos modelos ARMA e de curva de Phillips. Vale ressaltar que nem sempre esse é o caso.

2 Para detalhes sobre o processo de estimação e previsão com modelos ARMA, ver Box e Jenkins (1970). 
Os modelos VAR tornam-se super parametrizados rapidamente e, portanto, há uma troca entre expandir o conjunto de informação e parcimônia nesse tipo de modelo. Um modelo $\operatorname{VAR}(\mathrm{p})$ pode ser expresso como:

$$
y_{t}=c+\sum_{i=1}^{p} \Phi_{i} y_{t-i}+\varepsilon_{t}
$$

na qual, $y_{t}$ representa um vetor ( $\left.\mathrm{nx} 1\right)$ com valores das $n$ variáveis inclusas no modelo, e $p$ representa o número de defasagens das mesmas. Ou seja, em um VAR simétrico, como o utilizado neste estudo, todas as variáveis compõem todas as equações com o mesmo número de defasagens. Aqui, as variáveis utilizadas no modelo VAR foram PIB, inflação e taxa de juros. A escolha dessas variáveis é fundamentada na teoria econômica, bem como são encontradas frequentemente nos trabalhos sobre previsão de inflação.

\subsubsection{Modelos de Curva de Phillips}

No modelo de Phillips (1958) um excesso de oferta de trabalho na economia reduz a taxa de crescimento dos níveis de preços da economia. Quando a economia apresenta maiores taxas de inflação, os salários reais tornam-se menores, estimulando as firmas a contratar mais mão de obra. Portanto, quanto maior o desemprego menor é a taxa de inflação e vice-versa. O trabalho inicial de Phillips foi ampliado com a introdução das expectativas de inflação pelos trabalhos de Phelps $(1967,1969)$ e Friedman $(1968,1977)$. Então, uma redução do desemprego gera aumento da taxa de inflação, mas se este aumento for maior do que o esperado os trabalhadores passam a negociar os salários com base nessa expectativa, fazendo com que os salários reais e, consequentemente, a taxa de desemprego voltem aos seus níveis iniciais.

Entretanto, a hipótese aceleracionista de Friedman e Phelps implica em uma Curva de Phillips vertical no longo prazo, além de tornar esse modelo pouco atrativo para explicar a dinâmica de curto prazo entre inflação e atividade econômica. Caso as expectativas sejam racionais, a curva de Phillips será horizontal tanto no curto como no longo prazo (Sargent; Wallace, 1975). Mas, se as expectativas forem adaptativas, essa curva tende 
à horizontalidade. Esse comportamento dicotômico foi acompanhado de uma discussão dos fundamentos microeconômicos da relação entre essas variáveis. Nos trabalhos de Taylor (1980) e Calvo (1983), as flutuações da demanda agregada podem afetar variáveis nominais e reais da economia dependendo de como os contratos salariais e as expectativas forem formadas. Utilizando uma curva de salários similar a de Blanchflower e Oswald (1994), Roberts (1997) deriva uma Curva de Phillips com erros na forma de média móvel. Apesar dessa não ser a principal contribuição do trabalho de Roberts (1997), esse resultado aponta para a possibilidade de modelagens ARMA dos resíduos da Curva de Phillips serem necessárias.

Nos trabalhos de Gáli e Gertler (1999) e Gáli (2011), a Curva de Phillips é derivada a partir de modelos de equilíbrio geral com otimização dinâmica e o custo marginal da produção é utilizado no lugar das medidas usuais de hiato do produto. Ao permitir que a inflação dos produtos e insumos importados afete o custo marginal de produção, Choi e Cook (2008) propõem um modelo no qual os efeitos nominais das flutuações da taxa de câmbio são repassados para a inflação, formalizando resultados de Obstfeld e Rogoff (1995) sob a abordagem dos modelos novos Keynesianos. A partir desse ponto, vários trabalhos empíricos, como o de Burtstein, Eichenbaum e Rebelo (2005, 2006), e teóricos, como os de Zamulin e Styrin (2012) investigam os canais de transmissão dos repasses cambiais para os preços.

Os modelos baseados na Curva de Phillips utilizados neste trabalho podem ser expressos a partir da seguinte equação:

$$
\pi_{t}^{L}=\alpha_{1} \pi_{t-m}+\alpha_{2} h_{t-m}+\alpha_{3}\left(\Delta e_{t-0}+\pi_{t-0}^{*}\right)+\varepsilon_{t}
$$

A equação anterior é denominada de Curva de Phillips ampliada com repasse cambial. Nela a inflação depende da inflação passada (expectativa de inflação adaptativa ${ }^{3}$ ), do hiato do produto e do repasse cambial defasado. Outras versões mais simples dessa equação também são investigadas. Estima-se, inicialmente, a Curva de Phillips como função da inflação passada e do hiato do produto apenas. Posteriormente, realizam-se novas estimações com o repasse cambial inserido na equação. Depois, são estimadas fun-

3 Assim como todos os trabalhos internacionais e nacionais, exceto o de Ferreira (2014), sobre o uso da Curva de Phillips para previsão da inflação citados neste estudo, este trabalho não utiliza versões desse modelo com expectativas racionais. 
ções de transferência que combinam os modelos de Curva de Phillips com a modelagem ARMA de seus resíduos, as quais são representadas como:

$$
\begin{aligned}
& \pi_{t}^{L}=\alpha_{1} \pi_{t-m}+\alpha_{2} h_{t-m}+\alpha_{3}\left(\Delta e_{t-0}+\pi_{t-0}^{*}\right)+\phi(L) \theta(L) \varepsilon_{t} \\
& \phi(L)=\left(1-\phi_{1} L-\ldots-\phi_{p} L^{p}\right) \\
& \theta(L)=\left(1-\theta_{1} L-\ldots-\theta_{q} L^{q}\right)
\end{aligned}
$$

As equações (4.1) e (4.2) representam, respectivamente, os polinômios autorregressivos e de média móvel do modelo ARMA para os resíduos da equação (4). Em resumo, são estimados modelos $\operatorname{ARMA}(p, q)$, modelos VAR(p), modelos da Curva de Phillips simples, ampliada (com repasse cambial) e com modelos ARMA para seus resíduos. Todos esses modelos foram estimados e utilizados para a previsão utilizando as possíveis combinações com $m=1,2,3,4, n=1,2,3,4, o=0,1,2,3,4, p=0,1,2,3,4$ e $q=0,1,2,3,4$, dada a frequência trimestral dos dados. Assim, foram estimados 104 modelos para cada país da amostra, ou seja, 1.352 modelos.

\section{Dados}

A amostra utilizada é composta por dados de países desenvolvidos e de países em desenvolvimento. Os países desenvolvidos analisados são os integrantes do grupo G7: Alemanha, Canadá, EUA, França, Itália, Japão e Reino Unido. A amostra de países em desenvolvimento é formada por Brasil, Rússia, Índia, China, Uruguai e Argentina. Nesse último conjunto de países, estão os participantes que formam dois blocos econômicos denominados BRIC (Brasil, Rússia, Índia, China) e Mercosul ${ }^{4}$ (Brasil, Uruguai e Argentina).

O conjunto das variáveis utilizadas é formado pela taxa de inflação, PIB, taxa de juros e taxa de câmbio na frequência trimestral e foram extraídas do banco de dados do IPEADATA, FMI, OECD e IBGE. O período de tempo analisado consiste do primeiro trimestre de 1999 ao último trimestre de

4 O Mercosul é composto por Brasil, Uruguai, Argentina e Paraguai. Entretanto, por falta de informações em algumas variáveis utilizadas neste estudo, este último país foi retirado da amostra. 
2015, exceto nos dados da Argentina, Uruguai e Índia. No caso da Argentina e do Uruguai, a amostra é formada pelo primeiro trimestre de 1999 até o último trimestre de 2010, e na Índia os dados estão disponíveis até o último trimestre de $2014 .{ }^{5}$ As Tabelas 1 e 2 apresentam as informações sobre a base de dados utilizada.

Tabela 1 Informações sobre a base de dados: países desenvolvidos

\begin{tabular}{|c|c|c|}
\hline País & Variável & Fonte dos Dados \\
\hline \multirow[t]{5}{*}{ Alemanha } & PIB & Fundo Monetário Internacional- FMI \\
\hline & IPC & Fundo Monetário Internacional- FMI \\
\hline & Deflator & Fundo Monetário Internacional- FMI \\
\hline & Taxa de câmbio & Fundo Monetário Internacional- FMI \\
\hline & Taxa de juros & Banco Central \\
\hline \multirow[t]{5}{*}{ Canadá } & PIB & Fundo Monetário Internacional- FMI \\
\hline & IPC & Fundo Monetário Internacional- FMI \\
\hline & Deflator & Fundo Monetário Internacional- FMI \\
\hline & Taxa de câmbio & Fundo Monetário Internacional- FMI \\
\hline & Taxa de juros & Banco Central \\
\hline \multirow[t]{5}{*}{ EUA } & PIB & Fundo Monetário Internacional- FMI \\
\hline & IPC & Fundo Monetário Internacional- FMI \\
\hline & Deflator & Fundo Monetário Internacional- FMI \\
\hline & Taxa de câmbio & Fundo Monetário Internacional- FMI \\
\hline & Taxa de juros & Banco Central \\
\hline \multirow[t]{5}{*}{ França } & PIB & Fundo Monetário Internacional- FMI \\
\hline & IPC & Fundo Monetário Internacional- FMI \\
\hline & Deflator & Fundo Monetário Internacional- FMI \\
\hline & Taxa de câmbio & Fundo Monetário Internacional- FMI \\
\hline & Taxa de juros & Banco Central \\
\hline \multirow[t]{5}{*}{ Itália } & PIB & Fundo Monetário Internacional- FMI \\
\hline & IPC & Fundo Monetário Internacional- FMI \\
\hline & Deflator & Fundo Monetário Internacional- FMI \\
\hline & Taxa de câmbio & Fundo Monetário Internacional- FMI \\
\hline & Taxa de juros & Banco Central \\
\hline
\end{tabular}

(continua)

5 A escolha dos períodos foi baseada na disponibilidade dos dados para os países que compõem a amostra. Buscou-se trabalhar com o mesmo número de observações em cada economia. Entretanto, uma série de mesmo tamanho de IPC para Argentina e de PIB para o Uruguai e para a Índia não estão disponíveis. 
Tabela 1 (continuação)

\begin{tabular}{l|rr}
\hline País & Variável & Fonte dos Dados \\
\hline Japão & PIB & Fundo Monetário Internacional- FMI \\
\cline { 2 - 3 } & IPC & Fundo Monetário Internacional- FMI \\
\cline { 2 - 3 } & Deflator & Fundo Monetário Internacional- FMI \\
\cline { 2 - 3 } & Taxa de câmbio & Fundo Monetário Internacional- FMI \\
\cline { 2 - 3 } & Taxa de juros & Banco Central \\
\hline Reino Unido & PIB & Fundo Monetário Internacional- FMI \\
\cline { 2 - 3 } & IPC & Fundo Monetário Internacional- FMI \\
\cline { 2 - 3 } & Deflator & Fundo Monetário Internacional- FMI \\
\cline { 2 - 3 } & Taxa de câmbio & Fundo Monetário Internacional- FMI \\
\cline { 2 - 3 } & Taxa de juros & Banco Central \\
\hline
\end{tabular}

Fonte: Elaboração própria.

As taxas de juros são as utilizadas pelos Bancos Centrais para política monetária. As taxas de câmbio são em relação ao dólar americano, exceto no caso dos EUA, no qual o câmbio representa o custo Dólar por Euro.

Inicialmente, o correlograma das séries foi utilizado para identificar as séries com sazonalidade, as quais foram submetidas ao filtro X12 para a remoção desse componente. A taxa de inflação foi calculada com base no Índice de Preço ao Consumidor ${ }^{6}$ (IPC) para todos os países. Utiliza-se o deflator implícito do PIB para obter os valores constantes dos PIB de todos os países da amostra.

Tabela 2 Informações sobre a base de dados: países em desenvolvimento

\begin{tabular}{|c|c|c|}
\hline País & Variável & Fonte dos Dados \\
\hline \multirow[t]{5}{*}{ Brasil } & PIB & Fundo Monetário Internacional- FMI \\
\hline & IPC & Fundo Monetário Internacional- FMI \\
\hline & Deflator & Fundo Monetário Internacional- FMI \\
\hline & Taxa de câmbio & Fundo Monetário Internacional- FMI \\
\hline & Taxa de juros & Banco Central \\
\hline \multirow[t]{6}{*}{ Rússia } & PIB & Fundo Monetário Internacional- FMI \\
\hline & IPC & www.iflation.eu \\
\hline & Deflator & Fundo Monetário Internacional- FMI \\
\hline & Taxa de câmbio & Fundo Monetário Internacional- FMI \\
\hline & Taxa de juros & Banco Central \\
\hline & & (continua) \\
\hline
\end{tabular}


Tabela 2 (continuação)

\begin{tabular}{|c|c|c|}
\hline País & Variável & Fonte dos Dados \\
\hline \multirow[t]{5}{*}{ Índia } & PIB & Fundo Monetário Internacional- FMI \\
\hline & IPC & Fundo Monetário Internacional- FMI \\
\hline & Deflator & Fundo Monetário Internacional- FMI \\
\hline & Taxa de câmbio & Fundo Monetário Internacional- FMI \\
\hline & Taxa de juros & Banco Central \\
\hline \multirow[t]{5}{*}{ China } & PIB & Fundo Monetário Internacional- FMI \\
\hline & IPC & Fundo Monetário Internacional- FMI \\
\hline & Deflator & Fundo Monetário Internacional- FMI \\
\hline & Taxa de câmbio & Fundo Monetário Internacional- FMI \\
\hline & Taxa de juros & Fundo Monetário Internacional- FMI \\
\hline \multirow[t]{5}{*}{ Uruguai } & PIB & Fundo Monetário Internacional- FMI \\
\hline & IPC & IPEADATA \\
\hline & Deflator & Fundo Monetário Internacional- FMI \\
\hline & Taxa de câmbio & IPEADATA \\
\hline & Taxa de juros & Banco Central \\
\hline \multirow[t]{5}{*}{ Argentina } & PIB & Fundo Monetário Internacional- FMI \\
\hline & IPC & IPEADATA \\
\hline & Deflator & Fundo Monetário Internacional- FMI \\
\hline & Taxa de câmbio & Fundo Monetário Internacional- FMI \\
\hline & Taxa de juros & Banco Central \\
\hline
\end{tabular}

Fonte: Elaboração própria.

Não foram encontrados dados referentes ao Produto Interno Bruto Real da Índia.

Neste caso, tornou-se necessário o uso do PIB a custo de fatores deflacionado.

O hiato do produto é calculado com o filtro de Hodrick-Prescott. A taxa de câmbio utilizada é a moeda local do país em comparação com o dólar, exceto a taxa de câmbio americana, a qual representa o custo dólar por euro. Vale lembrar que com a introdução do euro em 1999 como moeda oficial vigente na União Europeia, os dados referentes à taxa de câmbio dos países inseridos neste bloco só podem ser observados a partir de 1999.

\section{Resultados}

Inicialmente, os testes Dickey-Fuller Aumentado (ADF) e o de Phillips- 
-Perron (PP) são utilizados para testar a hipótese nula de raiz unitária. As defasagens da regressão auxiliar dos testes ADF são selecionadas com base no critério de informação de Schwarz. Para o conjunto dos países desenvolvidos, os resultados dos testes de raiz unitária estão apresentados na Tabela 3. A hipótese nula de raiz unitária foi rejeitada para as séries de inflação e hiato do produto de todos os países, ao nível de significância de $5 \%$. Os resultados desses testes para as variáveis PIB, taxa de juros e taxa de câmbio indicam a presença de uma raiz unitária, para todos os países. Nesses casos, depois de aplicar a primeira diferença, essas séries tornam-se estacionárias.

Tabela 3 Testes de Raiz Unitária

\begin{tabular}{|c|c|c|c|c|c|c|c|c|}
\hline & \multicolumn{2}{|c|}{ Alemanha } & \multicolumn{2}{|r|}{ Canadá } & \multicolumn{2}{|c|}{ EUA } & \multicolumn{2}{|r|}{ França } \\
\hline & $\begin{array}{l}\text { t-sta- } \\
\text { tistic }\end{array}$ & Prob & $\begin{array}{l}\text { t-sta- } \\
\text { tistic }\end{array}$ & Prob & $\begin{array}{l}\text { t-sta- } \\
\text { tistic }\end{array}$ & Prob & $\begin{array}{l}\text { t-sta- } \\
\text { tistic }\end{array}$ & Prob \\
\hline PIB & $-1,9590$ & 0,6124 & $-2,7600$ & 0,2173 & $-1,9069$ & 0,6398 & $-2,2405$ & 0,4597 \\
\hline Inflação & $-7,5266$ & 0,0000 & $-10,507$ & 0,0000 & $-9,4925$ & 0,0000 & -3.8372 & 0,0210 \\
\hline Taxa de juros & $-1,5000$ & 0,5274 & $-3,0350$ & 0,1308 & $-1,9526$ & 0,3068 & $-1,5000$ & 0,5274 \\
\hline \multirow[t]{3}{*}{ Taxa de câmbio } & $-1,1391$ & 0,9140 & $-1,4183$ & 0,5682 & $-1,3785$ & 05875 & $-1,1391$ & 0,9140 \\
\hline & & & \multicolumn{2}{|r|}{ Itália } & \multicolumn{2}{|c|}{ Japão } & \multicolumn{2}{|c|}{ Reino Unido } \\
\hline & & & $\begin{array}{l}\text { t-sta- } \\
\text { tistic }\end{array}$ & Prob & $\begin{array}{l}\text { t-sta- } \\
\text { tistic }\end{array}$ & Prob & $\begin{array}{l}\text { t-sta- } \\
\text { tistic }\end{array}$ & Prob \\
\hline PIB & & & $-1,9656$ & 0,6087 & $-1,3983$ & 0,8527 & $-1,9705$ & 0,6059 \\
\hline Inflação & & & $-4,9600$ & 0,0007 & $-7,7600$ & 0,0000 & $-9,2770$ & 0,0000 \\
\hline Taxa de juros & & & $-1,5000$ & 0,5274 & $-1,4433$ & 0,1378 & $-2,4698$ & 0,3417 \\
\hline Taxa de câmbio & & & $-1,1391$ & 0,9140 & $-1,8011$ & 0,3768 & -1.5541 & 0.7998 \\
\hline
\end{tabular}

Fonte: elaboração própria.

Para os países em desenvolvimento, os resultados dos testes de raiz unitária estão apresentados na Tabela 4. Nessa tabela, verifica-se que a variável inflação pode ser considerada estacionária ao nível de $5 \%$ para todos os países analisados. Já para a variável PIB, a hipótese nula não foi rejeitada para nenhum dos países. $\mathrm{Na}$ análise da taxa de juros, observa-se a não rejeição da hipótese nula de raiz unitária para os países do Brasil, Rússia e Índia. Por fim, consoante à taxa de câmbio, somente as séries do Uruguai e Argentina apresentaram um comportamento estacionário a um nível de 5\% de significância. Nesse caso, aplicou-se a primeira diferença, após 
a qual os testes mencionados anteriormente rejeitam a hipótese nula de raiz unitária.

Tabela 4 Teste de raiz unitária para países em desenvolvimento

\begin{tabular}{l|rr|r|r|r|r}
\hline & & Brasil & & Rússia & & Índia \\
\cline { 2 - 8 } & t-statistic & Prob & t-statistic & Prob & t-statistic & Prob \\
\hline Inflação & $-3,362$ & 0,016 & $-6,499$ & 0,000 & $-7,344$ & 0,000 \\
\hline PIB & $-0,772$ & 0,963 & $-2,274$ & 0,441 & $-1,615$ & 0,777 \\
\hline Juros & $-2,084$ & 0,252 & $-2,961$ & 0,151 & $-2,328$ & 0,413 \\
\hline Câmbio & $-0,417$ & 0,985 & 0,095 & 0,997 & $-0,582$ & 0,977 \\
\hline & \multicolumn{7}{c}{ China } & & Uruguai & & Argentina \\
\cline { 2 - 8 } & t-statistic & Prob & t-statistic & Prob & t-statistic & Prob \\
\hline Inflação & $-4,788$ & 0,001 & $-3,962$ & 0,003 & $-4,684$ & 0,000 \\
\hline PIB & $-2,762$ & 0,216 & $-0,004$ & 0,995 & $-0,519$ & 0,980 \\
\hline Juros & $-5,129$ & 0,000 & $-3,799$ & 0,005 & $-2,928$ & 0,048 \\
\hline Câmbio & $-1,798$ & 0,695 & $-5,485$ & 0,000 & $-6,034$ & 0,000 \\
\hline
\end{tabular}

Fonte: elaboração própria.

A amostra total (1999.T1 - 2015.T4) é separada em dois períodos. Os 104 modelos para cada país da amostra, que totalizam 1.352 modelos, são estimados utilizando as observações de 1999T1 a 2007T4. Depois, previsões de um passo à frente são feitas para o período 2008T1-2015T47. Como medida de eficiência preditiva dos modelos é utilizado o erro de previsão ao quadrado médio (EQM). As próximas duas subseções apresentam os resultados obtidos para os países desenvolvidos e depois para os em desenvolvimento.

\subsection{Resultados para os países desenvolvidos}

Os modelos de Curva de Phillips neste arrtigo são denominados de CP $(m, n, o)$, em que $m, n$ e $o$ referem-se às defasagens da inflação passada, do hiato do produto e da taxa de câmbio, respectivamente. Assim como a

7 Para os países da Argentina e Uruguai, os modelos são estimados para o período 1999T1 a 2007T4, e as previsões realizadas para o período 2008T1-2010T4. Já para a Índia, os modelos são estimados para o período 1999T1 a 2007T4, e as previsões realizadas para o período 2008T1-2014T4. 
maioria dos trabalhos sobre previsão, os resultados do processo de estimação dos 52 modelos escolhidos entre os 1.352 modelos estimados não são apresentados, e a comparação entre os mesmos é realizada pelos seus erros de previsão médios ao quadrado.

A Tabela 5 apresenta os erros quadráticos médios das previsões (EOM) da taxa de inflação geradas por cada modelo estimado - ARMA, VAR e as variações da Curva de Phillips - para os países que compõem o G7, bem como a especificação do modelo (defasagens e variáveis utilizadas nas Curvas de Phillips). O modelo que apresenta o menor EQM é considerado o mais eficiente para prever a variável em questão e está destacado em negrito. De acordo com a Tabela 5, pode-se perceber que os modelos ARMA apresentaram menor EQM em comparação com os modelos da Curva de Phillips e VAR para os países Alemanha, Canadá, França, Itália, Japão e Reino Unido. Nos Estados Unidos, a Curva de Phillips é o modelo que gera previsões mais eficientes de acordo com o critério adotado.

Tabela 5 Resultados para os países desenvolvidos : ARMA, VAR, Curva de Phillips

\begin{tabular}{|c|c|c|c|}
\hline País & Melhor ARMA & Melhor VAR & $\begin{array}{r}\text { Melhor Curva de Phillips } \\
\text { com ARMA }\end{array}$ \\
\hline Alemanha & $\begin{array}{r}\operatorname{ARMA}(4,4) ; \\
E Q M=0,00001046\end{array}$ & $\begin{array}{r}\text { VAR1; } \\
\text { EQM=0,00001158 }\end{array}$ & $\begin{array}{r}C P(4,4,1) ; \\
E Q M=0,00001071\end{array}$ \\
\hline Canadá & $\begin{array}{r}\operatorname{ARMA}(4,4) ; \\
\mathrm{EQM}=0,00002441\end{array}$ & $\begin{array}{r}\text { VAR2; } \\
\mathrm{EQM}=0,00002762\end{array}$ & $\begin{array}{r}C P(4,2,3) \text {; } \\
E Q M=0,00002737\end{array}$ \\
\hline EUA & $\begin{array}{r}\operatorname{ARMA}(4,4) \\
\mathrm{EQM}=0,00005627\end{array}$ & $\begin{array}{r}\text { VAR2; } \\
\mathrm{EQM}=0,00007194\end{array}$ & $\begin{array}{r}C P(4,2,1) \\
E Q M=0,00005357\end{array}$ \\
\hline França & $\begin{array}{r}\operatorname{ARMA}(4,4) \\
\mathrm{EQM}=0,00001338\end{array}$ & $\begin{array}{r}\text { VAR1; } \\
\text { EQM=0,00003025 }\end{array}$ & $\begin{array}{r}C P(4,1,1) ; \\
E Q M=0,00002100\end{array}$ \\
\hline Itália & $\begin{array}{r}\operatorname{ARMA}(1,3) \\
\mathrm{EQM}=0,00001576\end{array}$ & $\begin{array}{r}\text { VAR4; } \\
\mathrm{EQM}=0,00001746\end{array}$ & $\begin{array}{r}\mathrm{CP}(1,2,1) ; \\
\mathrm{EQM}=0,00001726\end{array}$ \\
\hline Japão & $\begin{array}{r}\operatorname{ARMA}(4,4) ; \\
\mathrm{EQM}=0,00003411\end{array}$ & $\begin{array}{r}\text { VAR1; } \\
\mathrm{EQM}=0,00004564\end{array}$ & $\begin{array}{r}\mathrm{CP}(1,1,0) \text {; } \\
\mathrm{EQM}=0,00004192\end{array}$ \\
\hline Reino Unido & $\begin{array}{r}\operatorname{ARMA}(4,4) ; \\
\mathrm{EQM}=0,00002847\end{array}$ & $\begin{array}{r}\text { VAR4; } \\
\mathrm{EQM}=0,00005331\end{array}$ & $\begin{array}{r}C P(4,4,1) ; \\
E Q M=0,00003535\end{array}$ \\
\hline
\end{tabular}

Fonte: elaboração própria.

0 modelo que apresenta o menor EQM está destacado em negrito.

$C P(m, n, o): m, n$ e o são as defasagens da inflação passada, do hiato do produto e da taxa de câmbio, respectivamente. 
Com a introdução do componente ARMA na Curva de Phillips, observa-se uma mudança significativa nos resultados, como mostra a Tabela 6. Nessa tabela, CP(4,4,1):ARMA(4,4), significa, por exemplo, uma Curva de Phillips com quatro defasagens para a inflação, quatro defasagens para $\mathrm{O}$ hiato do produto e uma defasagem da taxa de câmbio, combinado com um modelo autorregressivo $\operatorname{ARMA}(4,4)$ para os resíduos da regressão. A simples introdução de termos ARMA nos resíduos da Curva de Phillips muda o ordenamento dos melhores modelos para prever a taxa de inflação. A Tabela 6 mostra que, exceto nos Estados Unidos, esse modelo gera previsões com menores EQM nos demais casos.

Tabela 6 Resultados para os países desenvolvidos: ARMA, VAR, Curva de Phillips e Função Transferência

\begin{tabular}{|c|c|c|c|}
\hline País & Melhor ARMA & Melhor VAR & $\begin{array}{r}\text { Melhor Curva de Phillips } \\
\text { com ARMA }\end{array}$ \\
\hline Alemanha & $\begin{array}{r}\text { ARMA }(4,4) ; \\
\text { EQM }=0,00001046\end{array}$ & $\begin{array}{r}\text { VAR1; } \\
\mathrm{EQM}=0,00001158\end{array}$ & $\begin{array}{r}\mathrm{CP}(4,4,1): \operatorname{ARMA}(4,4) ; \\
\mathrm{EQM}=0,00000829\end{array}$ \\
\hline Canadá & $\begin{array}{r}\text { ARMA }(4,4) \\
\text { EQM }=0,00002441\end{array}$ & $\begin{array}{r}\text { VAR2; } \\
\mathrm{EQM}=0,00002762\end{array}$ & $\begin{array}{r}\mathrm{CP}(4,2,3): \mathrm{MA}(4) ; \\
\mathrm{EQM}=0,00002306\end{array}$ \\
\hline EUA & $\begin{array}{r}\text { ARMA }(4,4) ; \\
\text { EQM }=0,00005627\end{array}$ & $\begin{array}{r}\text { VAR2; } \\
\mathrm{EQM}=0,00007194\end{array}$ & $\begin{array}{r}\mathrm{CP}(4,2,1): \mathrm{MA}(4) ; \\
\mathrm{EQM}=0,00005241\end{array}$ \\
\hline França & $\begin{array}{r}\text { ARMA }(4,4) ; \\
\text { EQM }=0,00001338\end{array}$ & $\begin{array}{r}\text { VAR1; } \\
\text { EQM }=0,00003025\end{array}$ & $\begin{array}{r}\mathrm{CP}(4,1,1): \operatorname{ARMA}(4,4) \\
\mathrm{EQM}=0,00001787\end{array}$ \\
\hline Itália & $\begin{array}{r}\text { ARMA(1,3); } \\
\text { EQM }=0,00001576\end{array}$ & $\begin{array}{r}\text { VAR4; } \\
\text { EQM }=0,00001746\end{array}$ & $\begin{array}{r}\mathrm{CP}(1,2,1): \mathrm{ARMA}(4,4) ; \\
\mathrm{EQM}=0,00001182\end{array}$ \\
\hline Japão & $\begin{array}{r}\operatorname{ARMA}(4,4) ; \\
\mathrm{EQM}=0,00003411\end{array}$ & $\begin{array}{r}\text { VAR1; } \\
\text { EQM }=0,00004564\end{array}$ & $\begin{array}{r}\mathrm{CP}(1,1,0): \mathrm{ARMA}(4,4) ; \\
\mathrm{EQM}=0,00003275\end{array}$ \\
\hline Reino Unido & $\begin{array}{r}\text { ARMA }(4,4) \\
\text { EQM }=0,00002847\end{array}$ & $\begin{array}{r}\text { VAR4; } \\
\text { EQM }=0,00005331\end{array}$ & $\begin{array}{r}\mathrm{CP}(4,4,1): \operatorname{ARMA}(4,4) ; \\
\mathrm{EQM}=0,00002840\end{array}$ \\
\hline
\end{tabular}

Fonte: Elaboração própria.

O modelo que apresenta o menor EQM está destacado em negrito.

$C P(m, n, o): m, n$ e o são as defasagens da inflação passada, do hiato do produto e da taxa de câmbio, respectivamente.

A Tabela 7 mostra que o uso de componentes ARMA nos resíduos da Curva de Phillips pode diminuir consideravelmente seus EOMs em todos os casos. Por exemplo, no caso da Itália, o EOM da Curva de Phillips com termos ARMA é $-32 \%$ menor do que o EOM da curva de Phillips sem esses termos. 
Tabela 7 Comparação dos EQM dos modelos de Curva de Phillips - países desenvolvidos

\begin{tabular}{l|r}
\hline País & $\Delta \%$ \\
\hline Alemanha & $-23 \%$ \\
\hline Canadá & $-16 \%$ \\
\hline EUA & $-22 \%$ \\
\hline França & $-15 \%$ \\
\hline Itália & $-32 \%$ \\
\hline Japão & $-22 \%$ \\
\hline Reino Unido & $-20 \%$ \\
\hline
\end{tabular}

Fonte: elaboração própria.

$\Delta \%=\left(E Q M C P^{A R M A}-E Q M C P / E Q M C P\right) \times 100$

$E Q M C P A R M A=E Q M$ da curva de Phillips com ARMA

$E Q M C P=E Q M$ da curva de Phillips sem ARMA

\subsection{Resultados para os países em desenvolvimento}

A Tabela 8 mostra que, de forma análoga ao caso anterior, os modelos ARMA geram as melhores previsões da inflação para essa amostra de países em desenvolvimento quando comparadas com as previsões geradas por modelos VAR e de Curva de Phillips sem efeitos ARMA nos seus resíduos.

Tabela 8 Resultados para os países em desenvolvimento: ARMA, VAR, Curva de Phillips

\begin{tabular}{|c|c|c|c|}
\hline País & Melhor ARMA & Melhor VAR & Melhor Curva de Phillips \\
\hline Brasil & $\begin{array}{r}\operatorname{ARMA}(4,1) ; \\
\mathrm{EQM}=0,00002626\end{array}$ & $\begin{array}{r}\text { VAR1; } \\
\mathrm{EQM}=0,00004646\end{array}$ & $\begin{array}{r}C P(1,3,0) \text {; } \\
E Q M=0,00004003\end{array}$ \\
\hline Rússia & $\begin{array}{r}\text { ARMA(4,4); } \\
\text { EQM=0,00013354 }\end{array}$ & $\begin{array}{r}\text { VAR1; } \\
E Q M=0,0001829\end{array}$ & $\begin{array}{r}C P(1,2,1) ; \\
E Q M=0,0001527\end{array}$ \\
\hline Índia & $\begin{array}{r}\operatorname{ARMA}(4,4), \\
\mathrm{EQM}=0,0001656\end{array}$ & $\begin{array}{r}\text { VAR 4; } \\
E Q M=0,0003592\end{array}$ & $\begin{array}{r}\mathrm{CP}(4,2,1) ; \\
\mathrm{EQM}=0,0001662\end{array}$ \\
\hline China & $\begin{array}{r}\text { ARMA(4,3), } \\
E Q M=0,00005062\end{array}$ & $\begin{array}{r}\text { VAR1; } \\
\mathrm{EQM}=0,00010595\end{array}$ & $\begin{array}{r}C P(1,1,4) ; \\
E Q M=0,0000956\end{array}$ \\
\hline Uruguai & $\begin{array}{r}\operatorname{ARMA}(2,1), \\
E Q M=0,0000840\end{array}$ & $\begin{array}{r}\text { VAR1; } \\
E Q M=0,0000499\end{array}$ & $\begin{array}{r}C P(1,2,4) ; \\
E Q M=0,0000546\end{array}$ \\
\hline Argentina & $\begin{array}{r}\operatorname{ARMA}(3,3), \\
\mathrm{EQM}=0,0000141\end{array}$ & $\begin{array}{r}\text { VAR1; } \\
E Q M=0,0000475\end{array}$ & $\begin{array}{r}C P(1,2,0) \text {; } \\
E Q M=0,0000230\end{array}$ \\
\hline
\end{tabular}

Fonte: elaboração própria. 
O modelo que apresenta o menor EQM está destacado em negrito.

$C P(m, n, o): m, n$ e o são as defasagens da inflação passada, do hiato do produto e da taxa de câmbio, respectivamente.

De maneira similar ao observado na amostra de países desenvolvidos, a Tabela 9 mostra que há uma melhora na eficiência preditiva dos modelos de Curva de Phillips ao se introduzir termos ARMA em seus resíduos, capaz de alterar quase completamente o rank dos melhores modelos da Tabela 8. A comparação dessas tabelas mostra que os modelos ARMA que apresentavam menores EQM para todos os países dessa amostra passam a apresentar menor EQM em apenas um caso (Brasil). Nos demais casos, a introdução dos termos ARMA nos resíduos das curvas de Phillips faz com que esses modelos gerem previsões com menores EQM.

Tabela 9 Resultados para os países em desenvolvimento: ARMA, VAR, Curva de Phillips

\begin{tabular}{|c|c|c|c|}
\hline País & Melhor ARMA & Melhor VAR & $\begin{array}{r}\text { Melhor Curva de Phillips } \\
\text { com ARMA }\end{array}$ \\
\hline Brasil & $\begin{array}{r}\text { ARMA(4,1); } \\
\text { EQM=0,00002626 }\end{array}$ & $\begin{array}{r}\text { VAR1; } \\
\text { EQM }=0,00004646\end{array}$ & $\begin{array}{r}\mathrm{CP}(1,3,0): \mathrm{ARMA}(4,1) ; \\
\mathrm{EQM}=0,0000283\end{array}$ \\
\hline Rússia & $\begin{array}{r}\text { ARMA }(4,4) ; \\
\text { EQM }=0,00013354\end{array}$ & $\begin{array}{r}\text { VAR1; } \\
\text { EQM }=0,0001829\end{array}$ & $\begin{array}{r}\text { CP(1,1,1):ARMA }(4,4) ; \\
\text { EQM=0,0001099 }\end{array}$ \\
\hline Índia & $\begin{array}{r}\text { ARMA }(4,4), \\
\text { EQM }=0,0001656\end{array}$ & $\begin{array}{r}\text { VAR 4; } \\
\text { EQM }=0,0003592\end{array}$ & $\begin{array}{l}\text { CP(1,3,4):AR(4); } \\
\text { EQM=0,0001464 }\end{array}$ \\
\hline China & $\begin{array}{r}\operatorname{ARMA}(4,3), \\
\mathrm{EQM}=0,00005062\end{array}$ & $\begin{array}{r}\text { VAR1; } \\
E Q M=0,00010595\end{array}$ & $\begin{array}{r}\mathrm{CP}(1,2,4): \mathrm{ARMA}(4,4) ; \\
\mathrm{EQM}=0,00004812\end{array}$ \\
\hline Uruguai & $\begin{array}{r}\operatorname{ARMA}(2,1), \\
\mathrm{EQM}=0,0000840\end{array}$ & $\begin{array}{r}\text { VAR1; } \\
E Q M=0,0000499\end{array}$ & $\begin{array}{r}\mathrm{CP}(1,2,4): \operatorname{ARMA}(2,1) ; \\
\mathrm{EQM}=0,0000193\end{array}$ \\
\hline Argentina & $\begin{array}{r}\operatorname{ARMA}(3,3), \\
\mathrm{EQM}=0,0000141\end{array}$ & $\begin{array}{r}\text { VAR1; } \\
\text { EQM }=0,0000475\end{array}$ & $\begin{array}{r}\mathrm{CP}(1,2,4): \operatorname{ARMA}(3,3) ; \\
\mathrm{EQM}=0,0000132\end{array}$ \\
\hline
\end{tabular}

Fonte: elaboração própria.

O modelo que apresenta o menor EQM está destacado em negrito

$C P(m, n, o): m$, n e o são as defasagens da inflação passada, do hiato do produto e da taxa de câmbio, respectivamente.

A Tabela 10 calcula as variações percentuais dos EQMs dos modelos de Curva de Phillips das Tabelas 8 e 9. Nessa tabela, verifica-se que os modelos da Curva de Phillips com termos ARMA reduzem os EQMs dos modelos sem estes processos na previsão da inflação da amostra de países 
em desenvolvimento, principalmente no caso do Uruguai e da China, nos quais a redução é aproximadamente de $-96 \%$ e $-50 \%$, respectivamente.

Tabela 10 Comparação dos EQMs dos modelos de Curva de Phillips - países em desenvolvimento

\begin{tabular}{l|r}
\hline País & $\Delta \%$ \\
\hline Brasil & $-29 \%$ \\
\hline Rússia & $-28 \%$ \\
\hline Índia & $-12 \%$ \\
\hline China & $-50 \%$ \\
\hline Uruguai & $-96 \%$ \\
\hline Argentina & $-42 \%$ \\
\hline
\end{tabular}

Fonte: elaboração própria.

$\Delta \%=(E Q M C P A R M A-E Q M C P / E Q M C P) \times 100$

$E Q M C P^{A R M A}=E Q M$ da curva de Phillips com ARMA

$E Q M C P=E Q M$ da curva de Phillips sem ARMA

\section{Conclusões}

O presente trabalho apresenta a comparação de previsões para a taxa de inflação trimestral de uma amostra de países desenvolvidos (economias que compõem o G7) e de outra amostra constituída de países em desenvolvimento, geradas por modelos baseados na Curva de Phillips sem e com uma simples introdução de uma modelagem ARMA de seus resíduos. O objetivo dessa comparação não é apenas verificar se modelos baseados na Curva de Phillips são bons previsores da inflação, mas também gerar evidências empíricas que respondam se uma simples especificação desse modelo, que inclua efeitos de média móvel, termos estes que são comuns na dinâmica de preços, deve ser usada como benchmark para estudos que propõem modelos econométricos ou de séries temporais mais elaboradas para prever essa variável.

Os resultados mostram que o uso de componentes ARMA nos resíduos da Curva de Phillips diminui consideravelmente seus erros quadrados médios de previsão (EQM) em todos os casos. Por exemplo, no caso da Itália, o EOM da Curva de Phillips com termos ARMA é -32\% menor do que o EOM da Curva de Phillips sem esses termos. Enquanto que no caso do 
Uruguai e da China essa variação é de $-96 \%$ e $-50 \%$, respectivamente.

$\mathrm{Na}$ amostra de países desenvolvidos, os modelos ARMA geram previsões mais eficientes do que as das Curvas de Phillips sem modelagem do resíduo e dos modelos VAR, exceto no caso dos Estados Unidos, no qual a Curva de Phillips apresentou melhores resultados. Tal conclusão é encontrada em vários estudos sobre previsão de inflação, como em Stock e Watson (2007) e Canova (2007). Entretanto, ao incluir a modelagem ARMA nos resíduos dos modelos de Curva de Phillips, a situação foi invertida em favor destes modelos que passaram a gerar previsões mais eficientes para quase todos os países desenvolvidos, sendo a França a única exceção.

Para a amostra dos países em desenvolvimento, a Curva de Phillips com termos ARMA para seus resíduos também melhora as previsões de inflação em relação aos modelos de Curva de Phillips sem esses componentes. Ao se comparar os resultados dos demais modelos ARMA e VAR, verifica-se que somente em uma das seis economias selecionadas para representar esse grupo as previsões geradas pelos modelos ARMA possuem os menores EQM.

Em geral, esses resultados evidenciam um possível ganho de eficiência preditiva dos modelos de Curva de Phillips ao se introduzir termos de média móvel ou autorregressivos em seus resíduos. Apesar dessa prática ser comum na previsão de outras variáveis e existir modelos teóricos que fundamentem a inclusão de componentes de média móvel na Curva de Phillips, as previsões desse tipo de modelo não são utilizadas na comparação da eficiência preditiva de modelos e técnicas alternativas para previsão da inflação. Portanto, uma extensão natural deste trabalho é verificar se modelos vetoriais Baysianos (BVAR), modelos DSGE e modelos de fatores dinâmicos podem gerar previsões para inflação com menores EQM do que modelos de Curva de Phillips com a simples introdução de processos de média móvel ou autorregressivos em seus erros.

\section{Referências}

AREOSA, W. D.; MEDEIROS, M. Inflation dynamics in brazil: the case of a small open economy. Brazilian Review of Econometrics, v. 27, n. 1, p. 131-166, 2007.

ARRUDA, E. F.; FERREIRA, R. T.; CASTELAR, I. Modelos lineares e não lineares da Curva de Phillips para previsão da taxa de inflação no Brasil. Revista Brasileira de Economia, v. 65, n. 3, p. 237-252, 2011. 
BOX, G; JENKINS, G. Time series analysis: Forecasting and control. San Francisco: Holdem-Day, 1970.

BLANCHFLOWER, D. G; OSWALD, A. J. The Wage Curve. Cidade?: Cambridge, MA, MIT Press, 1994.

BURSTEIN, A.; EICHENBAUM, M.; REBELO, S. Large Devaluations and the Real Ex-change Rate. Journal of Political Economy, 113 (4), p. 742-84, 2005.

BURSTEIN, A.; EICHENBAUM, M.; REBELO, S. The Importance of Nontradables Goods Prices in Cyclical Real Exchange Rate Fluctuations. Japan and the World Economy, 18 (3), p. 247-253, 2006.

CALVO, G. A. Staggered Prices in a Utility-Maximizing Framework. Journal of Monetary Economics, v. 12, p. 383-98, 1983.

CANOVA, F. G-7 inflation forecasts: random walk, phillips curve or what else?. Macroeconomic Dynamics, 11, 1-30, 2007.

CHOI, W. G; COOK, D. New Keynesian Exchange Rate Passthrough. IMF Working Paper, WP 08/213, 2008.

CORREA, A. S.; MINELLA, A. Mecanismos não-lineares de repasse cambial: um modelo de Curva de Phillips com threshold para o Brasil. XXXIII Encontro Nacional de Economia (ANPEC). Associação Nacional dos Centros de Pós-Graduação em Economia, 2005.

CYSNE, R. P. A relação de Phillips no Brasil: 1964-1966 versus 1980-1984. Revista Brasileira de Economia, v. 39, n. 4, 401-422.

FERREIRA, D. Essays in Nonlinear Macroeconometrics: Fiscal Policy and Phillips Curve. 2014. Dissertação (Mestrado em Desenvolvimento Econômico) - Universidade Federal do Paraná.

FERREIRA, D.; PALMA, A.A. Forecasting inflation with the Phillips curve: A dynamic model averaging approach for Brazil. Revista Brasileira de Economia, v. 69, n. 4, p. 451-465, 2015.

FRIEDMAN, M. The role of Monetary policy. American Economic Review, v. 58, p. 1-17, 1968.

FRIEDMAN, M. Inflation and Unemployment: Nobel lecture. Journal of Political Economy, 85, 1977.

GALI, J. The return of the wage Phillips curve. Journal of the European Economic Association, v. 9 , n. 3, p. 436-461, 2011.

GALI, J.; GERTLER, M. Inflation dynamics: A structural econometric analysis. Journal of monetary Economics, v. 44, n. 2, p. 195-222, 1999.

LIMA, E. J. A.; ARAUJO, F.; SILVA, J. R. C. Previsão e modelos macroeconômicos no banco central do Brasil. Banco Central do Brasil. Dez anos de metas para a inflação - 1999-2009. Brasília: Banco Central do Brasil, 2011. p. 353-399.

MAKA, A.; BARBOSA, F. H. Phillips curves: An encompassing test. XLI Encontro Nacional de Economia(ANPEC). Associação Nacional dos Centros de Pós-Graduação em Economia, 2013.

MAZALI, A. A.; DIVINO, J. A. Real wage rigidity and the new Phillips curve: The Brazilian case. Revista Brasileira de Economia, v. 64, n. 3, p. 291-306.

MENDONÇA, M.J.C.; SACHSIDA, A.; MEDRANO, L.A.T. Inflação versus desemprego: 
novas evidências para o Brasil. Economia Aplicada, v. 16, n. 3, p. 475-500, 2012.

OBSTFELD, M.; ROGOFF, K. Exchange rate dynamics redux. Journal of political economy, v. 103, n. 3, p. 624-660, 1995.

PHELPS, E. S. Phillips curve expectation of inflation and optimum unemployment over time. Economica, v. 34, p. 254-81, 1967.

PHELPS, E. S. The new microeconomic in inflation and employment theory. American Economic Review, v. 59, p. 147-160, 1969.

PHILLIPS, A. W. (1958). The Relationship between Unemployment and the Rate of Change of Money Wages in the United Kingdom 1861-1957. Economica, 25(100), p. 283-299, 1958.

PORTUGAL, M. S.; MADALOZZO, R. C. Um modelo de NAIRU para o Brasil. Revista de Economia Política, v.20, n.4, , p. 26-47, 2000.

RUMLER, F ; VALDERRAMA, M. T. Comparing the New Keynesian Phillips Curve with Time Series Models to Forecast Inflation. Working Paper Series From Oesterreichische National bank, n. 148, 2008.

ROBERTS, J. The wage curve and the Phillips curve. Federal Reserve Board, Finance and Economics Discussion Series WP, p. 97-57, 1997.

SACHSIDA, A. Inflação, desemprego e choques cambiais: uma revisão da literatura sobre a curva de Phillips no Brasil. Revista Brasileira de Economia, v. 67, n. 4, p. 549-559, 2013.

SACHSIDA, A.; RIBEIRO, M.; SANTOS, C. H. A curva de Phillips e a experiência brasileira (Texto para Discussão n. 1429). Instituto de Pesquisa Econômica Aplicada-IPEA, 2009

SARGENT, T. J; WALLACE, N. (1975). "Rational" expectations, the optimal mometary instrument, and the optimal money supply rule. Journal of Political Economy, 83, p. 241-254, 1975.

SCHWARTZMAN, F.F. Estimativa de curva de Phillips para o Brasil com preços desagregados. Economia Aplicada, v. 10, n. 1, p. 137-155, 2006.

STOCK, J.H.; WATSON, M. W. Forecasting inflation. Journal of Monetary Economics, v. 44, n. 2, p. 293-335, 1999.

STOCK, J. H.; WATSON, M. W.Forecasting Output and Inflation: The Role of Asset Prices. Journal of Economic Literature, v.41, n.3, p. 788-829, 2003.

STOCK, J. H.; WATSON, M. W. Why has U.S. inflation become harder to forecast?. Journal of Money, Credit and Banking, v. 39, n. 1, p. 3-33, 2007.

TAYLOR, J. B. Aggregate dynamics and staggered contracts. Journal of Political Economy, 88, p. 1-23, 1980.

ZAMULIN, O.; STYRIN, K. A real Exchange rate Phillips curve. Working Paper 0179, Center for Economic and Financial Research, 2012.

\section{Sobre os autores}

Erika Vanessa Alves da Silva - erika_vanessa01@hotmail.com

Universidade Federal do Ceará.

Nathália da Silva Oliveira -nathalialara19@hotmail.com

Universidade Federal do Ceará. 
Roberto Tatiwa Ferreira-rtf2@uol.com.br

Universidade Federal do Ceará.

Cristianoda Costa da Silva - cristiano.dacostadasilva@hotmail.com

Universidade do Estado do Rio Grande do Norte.

\section{Sobre 0 artigo}

Recebido em 11 de novembro de 2015. Aprovado em 25 de outubro de 2016. 